\title{
Congestive Heart Failure and Upper Digestive Endoscopic Lesions
}

\author{
Adriana-Stela Cosma¹, Claudia Bănescu², Simona Mocan³ ${ }^{3}$ Beáta Balla4, Anca Negovan ${ }^{*}$ \\ 1. Genetics Laboratory of the Emergency County Hospital, Gheorghe Marinescu 50, Târgu Mureș 540136, Mureș, Romania \\ 2. Department of Medical Genetics, University of Medicine, Pharmacy, Sciences and Technology of Târgu Mureș, Gheorghe Marinescu 38, Tîrgu Mureș \\ 540139 Mures, Romania \\ 3. Pathological Department, Emergency County Hospital Târgu Mures, Gheorghe Marinescu 50, 540136, Mures, Romania \\ 4. Genetics Laboratory of the Emergency County Hospital, Gheorghe Marinescu 50, Târgu Mureș 540136, Mureș, Romania \\ 5. University of Medicine, Pharmacy, Sciences and Technology of Tîrgu Mureș, Gheorghe Marinescu 38, Târgu Mureș 540139 Mures, Romania
}

Objective: To evaluate the impact of congestive heart failure and the most important clinical and pathological factors on severe upper digestive mucosal lesions. Methods: The study included 749 patients referred for upper digestive endoscopy, divided into two groups: 140 subjects with congestive heart failure (study group) and 609 subjects without heart failure (control group). Results: Severe endoscopic lesions quantified according to Lanza score (OR = 3.84, 95\% IC: 2.62-5.62), active/inactive gastritis (OR = 2.07, 95\% Cl: 1.36-3.14), intestinal metaplasia and/or gastric atrophy $(\mathrm{OR}=2.42,95 \% \mathrm{Cl}: 1.67-3.52)$ were significant more frequent among patients with heart failure. Anemia $(\mathrm{OR}=3.65,95 \% \mathrm{IC}: 2.48-5.37)$ and all investigated comorbidities, as well as alcohol consumption (OR = 1.60, 95\% IC: $1.10-2.34)$ and smoking $(\mathrm{OR}=1.76,95 \% \mathrm{IC}: 1.17-2.64)$ were more frequent in the study-group. Dividing the patients with cardiac insufficiency according to the severity of their endoscopic lesions, the male gender ( $O R=2.76,95 \%$ IC: $1.35-5.61)$ and daily low-dose aspirin consumption were found to be more frequent among patients with severe endoscopic lesions (OR =7.71, 95\% IC: $3.62-16.40)$, while anticoagulant therapy and alcohol consumption were borderline associated with mucosal lesions $(p=0.08)$. Conclusions: Male patients and aspirin consumers with heart failure, but not those with $\mathrm{H}$. pylori infection seem to be more prone to develop upper digestive endoscopic lesions, while alcohol consumption or anticoagulant therapy could be other modifiable factors associated with severe endoscopic lesions in a congestive gastro-duodenal mucosa.

Keywords: congestive heart failure, endoscopic gastro-duodenal lesions, anemia

Received 16 December 2018 / Accepted 14 January 2019

\section{Introduction}

Heart failure is a common and potentially fatal condition, being one of the most frequent causes of hospitalization today, with a poor prognosis despite the improvements in diagnosis and medical treatment [1,2]. In 2016 it affected over 60 million people worldwide and despite improvements in modern device-, and pharmacotherapy, it continues to have a high mortality [3].

Congestive heart failure (CHF) can be described as a multi-organ disorder caused by the incapacity of the heart to keep adequate cardiac output to satisfy the body's metabolic needs [4]. It has been established that the cardiovascular system is not the only one affected by heart failure. In $\mathrm{CHF}$, the increased systemic venous congestion is transmitted to the inferior vena cava, which leads to congestion in its draining territories, such as the gastrointestinal tract (GIT) mucosa [5]. Over the last decade, several studies investigated the gastrointestinal changes associated with CHF $[5,6]$. Structural changes have been previously described in the gastric mucosa, such as mosaic pattern in the stomach, mucosal thickening, antral vascular ectasia, and areas of telangiectasias [6].

Mechanisms of gastrointestinal-related symptoms remain poorly understood despite their common presence and increased morbidity and mortality correlated with their coexistence. The specific involvement of the gastrointestinal system in CHF results in a bidirectional relationship. The systemic volume overload characteristic of CHF is generally associated with concomitant gastrointestinal edema, which can result bacterial translocation into the systemic circulation. Consequent activation of monocytes and excessive release of cytokines lead to systemic inflammation, increased symptoms, and therefore, progression of the disease [4].

Anemia is a very common and well-known comorbidity in patients with $\mathrm{CHF}$ and its prevalence increases with the severity of the disease. The true frequency of anemia in CHF patients varies widely, but it has been reported to range between $30 \%-50 \%$, depending on the severity of $\mathrm{CHF}$ and the population studied $[6,7,8,9]$. Anemia in CHF patients is a multifactorial and multidimensional problem. However, there has been an increasing appreciation for the significance of anemia in the pathophysiology, treatment, and prognosis of CHF [9].

The aim of the present study is to evaluate the influence of $\mathrm{CHF}$ and associated clinical and pathological factors on the severity of upper digestive endoscopic lesions.

\section{Methods}

The study included 749 patients divided as follows: $140 \mathrm{pa}-$ tients with congestive heart failure (CHF group) and 609 
subjects without congestive heart failure (control group). All patients were hospitalized in Medical Clinic Nr. 3 in Tîrgu Mureș Emergency County Hospital, Romania, and underwent an upper digestive endoscopy. The reasons for endoscopy were specific digestive symptoms, anemia, or screening before initiating an antithrombotic therapy or a major cardiovascular surgery.

Written informed consent was obtained from all subjects before being included in the study. The research was approved by the Ethical Committee of the University of Medicine and Pharmacy of Tîrgu Mureș, Romania. Demographical and clinical data were collected from each patient after structured interviews and clinical examinations.

The diagnosis of CHF was derived from a careful history and based on present and past medical records of the patients. Digestive symptoms questioned were epigastric pain, heartburn, regurgitation, nausea/vomiting. Alcohol consumption was considered at the use of at least 10 units $(10 \mathrm{~mL})$ of pure alcohol weekly, while smoking at more than 5 cigarettes/day. To conduct an investigation into drug exposure, medical records of the patients and a structured interview was performed. Patients taking low-dose aspirin (LDA, $75-100 \mathrm{mg} /$ day), regular daily doses of non-aspirin non-steroidal anti-inflammatory drugs (NSAIDs-ibuprofen, ketoprofen, dexketoprofen, diclofenac) and proton pump inhibitors (omeprazole, pantoprazole, esomeprazole) for more than 2 weeks were considered as exposed to drug. Cut-off values of hemoglobin level for anemia (hemoglobin level of $<12 \mathrm{~g} / \mathrm{dl}(7.5 \mathrm{mmol} / \mathrm{l})$ in women and $<13 \mathrm{~g} / \mathrm{dl}(<8.1 \mathrm{mmol} / \mathrm{l})$ in men, $)$ were used according to the WHO definitions [7].

Each endoscopy was carried out by an endoscopist who was not informed about the symptoms and drug exposure. The mucosal lesions were described as erythema, petechiae (hemorrhagic area without mucosal defect), erosions (mucosal defect smaller than $5 \mathrm{~mm}$ ), or ulcers in both gastric and duodenal mucosa. We used the modified Lanza score (MLS), as followed: Lanza score 0 for no mucosal lesions, Lanza score 1 for one erosion or petechiae, Lanza score 2 for 2-10 erosions or petechiae, Lanza score 3 for more than 10 erosions or petechiae and Lanza score 4 when an ulcer was present [10]. To investigate factors associated with endoscopic lesions we stratified patients in CHF group according to their lesions in severe endoscopic lesions group (Lanza score 2,3,4 - 77 subjects) and controls (Lanza score 0,1- 63 subjects). The Los Angeles Classification System for the endoscopic assessment of reflux esophagitis was used to define esophagitis, but patients in the present study were assigned as having or not any mucosal lesions. During endoscopy, four biopsy specimens were taken, two from the antrum and two from the corpus (from the greater and the lesser curvature ). The specimens were routinely processed and examined by a pathologist blinded to symptoms and drug exposure. Mucosal changes in the gastric biopsies were described and classified using the Updated Sydney System. H. pylori infection was considered present if the germ was identified on histologic examination in at least one biopsy sample.

All collected data was recorded in a specially designed database.

\section{Statistical Analysis}

Qualitative nominal variables were summarized using absolute frequencies (number of cases) and relative frequencies (\%). Chi-square and Fisher's exact tests were performed to analyze the associations between possible predictors for congestive heart failure. Value of $p$ lower than 0.05 was considered statistically significant. The odds ratio (OR) and $95 \%$ confidence intervals were calculated to quantify the magnitude of the association. GraphPad Prism 6 was used for the statistical analysis.

\section{Results}

\section{Bivariate Analysis}

Distribution of demographic and clinical characteristics of the patients in two groups are showed in Table I. Male patients were more frequent in the CHF group. Anemia and severe endoscopic lesions were with statistically significant higher frequency in the $\mathrm{CHF}$ group $(\mathrm{OR}=3.65,95 \% \mathrm{IC}$ : 2.48-5.37), $(\mathrm{OR}=3.84,95 \%$ IC: $2.62-5.62)$. LDA and NSAIDs consumption was statistically lower among CHF patients compared with the control group $(\mathrm{OR}=0.41$, 95\% IC: 0.74-0.71).

Gastritis (active/inactive) $(\mathrm{OR}=2.07,95 \% \mathrm{CI}: 1.36$ $3.14)$, intestinal metaplasia and/or gastric atrophy in biopsy samples $(\mathrm{OR}=2.42,95 \% \mathrm{CI}: 1.67-3.52)$ were significantly associated with congestive heart failure, but not with active $\mathrm{H}$. pylori infection (Table I).

The history of ulcer, as well as concomitant diseases (respiratory, liver, renal, or cerebrovascular disease) were significantly more frequent in patients with congestive heart failure $(\mathrm{OR}=23.96,95 \% \mathrm{CI}: 14.75-38.94)$,. Consumption of gastrotoxic drugs (LDA and NSAIDs) was statistically significant less frequent among patients with CHF, while gastroprotective drugs (PPI) were more frequently taken. Epigastric pain and heartburn were found to be less frequent among patients with heart failure. Alcohol consumption (more than 2 units/day) and smoking (over 5 cigarettes/day) also showed significant association with congestive heart failure.

Dividing patients from CHF group according to the severity of endoscopic lesions (Lanza score) we observed that from all considered predictors, the male gender was positively associated with the severity of endoscopic lesions ( $\mathrm{OR}=2.76,95 \%$ IC: $1.35-5.61)$, while alcohol consumption had a tendency toward statistical significance (Table II). LDA consumption was found to be more frequent among patients with $\mathrm{CHF}$ and severe endoscopic lesions $(\mathrm{OR}=7.71,95 \%$ IC: 3.62-16.40), mean while anticoagulants tended to have a tendency toward significance. Anemia was more frequent in patients with CHF and severe 
Table I. The distribution of demographical, clinical, endoscopic and pathological variables in studied groups

\begin{tabular}{|c|c|c|c|c|c|c|c|}
\hline \multirow[t]{2}{*}{ Variables } & \multicolumn{2}{|c|}{$\begin{array}{l}\text { Congestive heart failure group } \\
\qquad N=140(18.70 \%)\end{array}$} & \multicolumn{2}{|c|}{$\begin{array}{c}\text { Control Group } \\
\mathrm{N}=609(81.30 \%)\end{array}$} & \multirow[t]{2}{*}{$\mathbf{p}^{*}$ value } & \multirow[t]{2}{*}{ OR } & \multirow[t]{2}{*}{$95 \% \mathrm{Cl}$} \\
\hline & $\mathrm{N}$ & $\%$ & $\mathrm{~N}$ & $\%$ & & & \\
\hline Male gender & 89 & 63.57 & 263 & 43.18 & $<0.0001$ & 2.29 & $1.57-3.35$ \\
\hline Anemia & 68 & 48.57 & 125 & 20.52 & $<0.0001$ & 3.65 & $2.48-5.37$ \\
\hline \multicolumn{8}{|l|}{ Drug consumption } \\
\hline Anticoagulants & 65 & 46.42 & 30 & 4.92 & $<0.0001$ & 16.73 & $10.20-27.44$ \\
\hline NSAIDs & 25 & 17.85 & 512 & 84.07 & $<0.0001$ & 0.04 & 0.02-0.06 \\
\hline PPIs & 83 & 59.28 & 285 & 46.79 & 0.0086 & 1.65 & $1.14-2.40$ \\
\hline LDAa & 17 & 12.14 & 151 & 24.79 & 0.0010 & 0.41 & $0.24-0.71$ \\
\hline \multicolumn{8}{|l|}{ Endoscopic findings } \\
\hline Severe endoscopic lesions & 77 & 55 & 147 & 24.13 & $<0.0001$ & 3.84 & $2.62-5.62$ \\
\hline Esophagitis & 30 & 21.42 & 141 & 23.15 & 0.73 & 0.90 & $0.57-1.41$ \\
\hline Biliary reflux & 48 & 34.28 & 218 & 35.79 & 0.76 & 0.93 & $0.63-1.37$ \\
\hline \multicolumn{8}{|l|}{ Histologic findings } \\
\hline Reactive gastropathy & 34 & 24.28 & 154 & 25.28 & 0.91 & 0.94 & $0.61-1.45$ \\
\hline Active/inactive gastritis & 106 & 75.71 & 366 & 60.09 & 0.0005 & 2.07 & $1.36-3.14$ \\
\hline $\mathrm{GA} / \mathrm{IMb}$ & 78 & 55.71 & 208 & 34.15 & $<0.0001$ & 2.42 & $1.67-3.52$ \\
\hline H. pylori infection & 47 & 33.57 & 220 & 36.12 & 0.62 & 0.89 & $0.60-1.31$ \\
\hline \multicolumn{8}{|l|}{ Comorbidities } \\
\hline Ulcer history & 81 & 57.85 & 33 & 5.41 & $<0.0001$ & 23.96 & $14.75-38.94$ \\
\hline Cerebrovascular disease & 13 & 8.66 & 15 & 2.46 & 0.001 & 3.75 & $1.74-8.08$ \\
\hline Renal disease & 64 & 45.71 & 41 & 6.73 & $<0.0001$ & 11.67 & $7.36-18.47$ \\
\hline Liver disease & 80 & 57.14 & 213 & 34.97 & $<0.0001$ & 2.47 & $1.70-3.60$ \\
\hline Respiratory disease & 69 & 49.28 & 77 & 12.64 & $<0.0001$ & 6.71 & $4.46-10.10$ \\
\hline Osteoarticular disease & 61 & 43.57 & 155 & 25.45 & $<0.0001$ & 2.26 & $1.54-3.30$ \\
\hline \multicolumn{8}{|l|}{ Symptoms } \\
\hline Epigastric pain & 61 & 43.57 & 367 & 60.26 & 0.0004 & 0.50 & $0.35-0.73$ \\
\hline Heartburn & 14 & 10 & 193 & 31.69 & $<0.0001$ & 0.23 & $0.13-0.42$ \\
\hline Regurgitation & 7 & 5 & 42 & 6.89 & 0.56 & 0.71 & $0.31-1.61$ \\
\hline Nausea/vomiting & 34 & 24.28 & 132 & 21.67 & 0.49 & 1.15 & $0.75-1.78$ \\
\hline \multicolumn{8}{|l|}{ Social behaviours } \\
\hline Alcohol consumptionc & 58 & 41.42 & 186 & 30.54 & 0.0162 & 1.60 & $1.10-2.34$ \\
\hline Smokingd & 45 & 32.14 & 129 & 21.18 & 0.0076 & 1.76 & $1.17-2.64$ \\
\hline
\end{tabular}

endoscopic lesions, in comparison with no-lesions group, but without statistical significance, while $H$. pylori infection was not associated with the severity of endoscopic lesions.

\section{Discussion}

Cardiovascular diseases (CV), including CHF, are the leading cause of morbidity and mortality worldwide. Physiologically, the gastrointestinal tract is one of the most intensely perfused organ, and mucosal gastric congestion is expected in CHF, due to the increase systemic venous pressure, which is transferred to the portal circulation through the hepatic venous bed $[6,11]$. Our results sustain that CHF by itself seems to be a factor influencing the severe gastro-duodenal endoscopic lesions, but not esophageal ones. Reactive gastropathy changes involve the congestion of superficial mucosal capillaries alongside with prominent mucin depletion, foveolar hyperplasia, and fibro-muscular replacement of the lamina propria. These changes appear after various type of aggressors, like alcohol consumption, biliary reflux and gastrotoxic drug consumption, having an impact on the balance of the gastric epithelium developing a constellation of mucosal changes [12]. In our study, the frequency of reactive gastropathy histologic changes were comparable in patients with and without $\mathrm{CHF}$ and were non-significant less frequent in patients with CHF and severe endoscopic lesions. Our results support the possible role of other aggressive factors $(H$. pylori infection, aging mucosa) on endoscopic lesions occurrence, not only the congestion in upper digestive tract.

Infection with $H$. pylori may be directly or indirectly involved in the pathogenesis of cardiovascular diseases. Altered iron metabolism is one of the leading mechanism of H. pylori, which can contribute to cardiovascular diseases [13]. In our study, $H$. pylori was not more frequent in patients diagnosed with congestive heart failure $(p=0.62)$. In a geographical area with a high prevalence of $H$. pylori infection, the histologic changes of gastric mucosa in elderly patients are usually related to early acquisition of infection. In our research, the inflammatory and premalignant histological gastric changes were more common in patients with heart failure $(\mathrm{OR}=2.42,95 \% \mathrm{IC}: 1.67-3.52)$ as they were older age than patients in the control group $(69.75 \pm 0.76$ years old for cases vs. $54.10 \pm 0.55$ years old for controls), but these findings seemed to not influence the frequency of endoscopic lesions. 
Table II: The distribution of studied variables in patients with CHF divided according to their endoscopic lesions

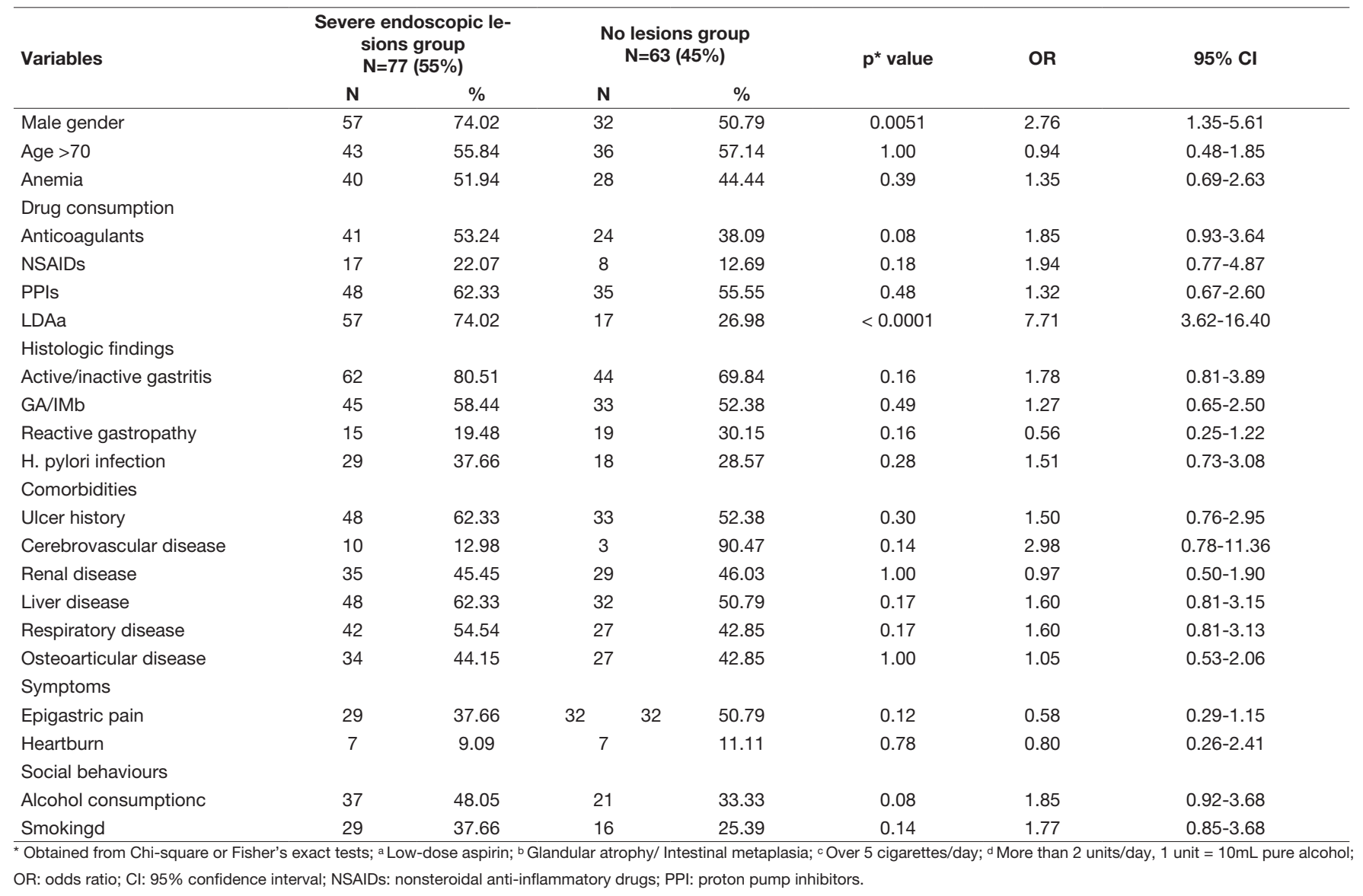

In our present study, anemia appeared to be more frequent in patients with CHF, but not in those with endoscopic lesions. The approach of the underlying mechanism of anemia in patients with CHF is very difficult, in most cases, more than one etiology is involved. Such factors are bone marrow dysfunction, renal dysfunction, abnormal steroid metabolism, hemodilution, resistance to erythropoietin, the use of drugs for the treatment of $\mathrm{CHF}$, chronic inflammation, hematinic deficiencies, decrease of food intake, reduction of intestinal absorption, and blood losses by the GIT $[6,7,8,9]$. Among anemic CHF patients the most frequent form of hematinic deficiency, besides folate and vitamin B12 deficiency, is represented by iron deficiency $[14,15,16]$. An other important factor which may induce anemia is chronic gastrointestinal blood loss. Many patients with CHF use antithrombotic treatments, antiplatelet and/or anticoagulants. These drugs promote the blood loss by the entire GIT from various mucosal lesions $[17,18,19,20]$. Furthermore, gastrointestinal conditions that do not usually induce bleeding, frequently associate iron deficiency anemia due to impairment of iron metabolism [21]. Our results support the important role of other combined mechanism except for bleeding from upper digestive endoscopic lesions in anemic patients with $\mathrm{CHF}$ that required a more complex approach.

The consumption of gastro-toxic drugs (NSAIDs, LDA), was less frequent in patients with CHF, while anticoagulants were more frequent, as the international thera- peutic guideline recommends their use in treatment or secondary prevention of the underlying conditions. Among all questioned variable, the aggressive effect of antiplatelet therapy (LDA), in a vulnerable congestive gastric mucosa was supported by our present and past results, while the role of anticoagulants should be further investigated in larger studies [22]. It has been demonstrated that daily LDA consumption reduces the risk of cardiovascular diseases, however, it also associates adverse effects, mostly in the gastrointestinal tract. These complications can range from mild upper events (dyspepsia, petechiae, or erosions) to severe events (peptic ulcer disease and bleeding) [23,24]. Based on present observations, antiplatelet therapy should be cautioned in patients diagnosed with $\mathrm{CHF}$, and gastroprotective therapy should be offered in high risk patients.

The presence of epigastric pain and heartburn were negatively associated with CHF, probably due to the selection of the cases: controls usually referred for symptoms, while cases for bleeding risk assessment. Different results were obtained in a study of 57 patients with congestive heart failure complaining from GI symptoms [5,11].

Our findings suggest that male gender was more frequently associated with $\mathrm{CHF}$ and severe lesions on endoscopy. In our previous research we obtained similar results regarding the severity of endoscopic lesions in patients consuming LDA [17]. Male gender presents cardiovascular diseases more commonly than females, due to hormonal differences [25]. On the other hand, they are more 
frequently affected by duodenal ulcer in relationship with H. pylori infection [26]. This association should be further questioned in larger studies adjusted for the most important confounding factors (smoking, alcohol consumption) $[27,28]$.

Patients suffering from CHF usually have at least one comorbidity and the severity of the heart failure leads to increasing numbers of comorbidities. The high number of comorbidities in our study was associated with the elderly population suffering from heart failure. Renal disease and anemia were the most common comorbidities found in a study conducted by van Deursen [29]. Similar results were found in our study, but the comorbidities did not appear to influence the severity of endoscopic lesions.

In this study, alcohol use was borderline correlated with endoscopic lesions, probably due to its additive aggressive effect on the gastrointestinal mucosa. Similar findings were described in a research that investigated bleeding in aspirin consumers [30]. Alcohol is considered to contribute to the development of cardiovascular diseases. Regular light alcohol drinking ( $<$ three drinks per day) may confer protective effects on heart failure associated with coronary heart disease. The protective effect disappears in heavy drinking (> three drinks per day) with an increases risks to develop cardiomyopathy, supraventricular arrhythmias, and systemic hypertension [31,32].

To the best of our knowledge this is the first study investigating histological and endoscopic upper digestive findings in a Romanian population with CHF. It's limitations are represented by the lack of regressions and adjustments based on confounding factors that will be approached in further studies. The present research questioning the impact of various demographical, clinical and histologic parameters on endoscopic lesions in patients with CHF may offer important clues for preventive strategy development in Romanian population, characterized by a high frequency of $H$. pylori infection and its consequences (ulcer, premalignant lesions, cancer).

\section{Conclusions}

Based on our findings, we can conclude that male patients and low-dose aspirin consumers with CHF, but not those with $H$. pylori infection seem to be more prone to develop upper digestive endoscopic lesions, while anticoagulants and alcohol consumption could be associated with severe endoscopic lesions in a congestive gastro-duodenal mucosa.

\section{Authors' contribution}

Adriana-Stela Cosma (Conceptualization; Data curation; Formal analysis; Writing -original draft)

Claudia Bănescu (Conceptualization; Data curation; Methodology; Supervision; Validation; Writing - review \& editing)

Simona Mocan (Investigation; Validation)

Beáta Balla (Investigation; Writing - review \& editing)
Anca Negovan (Conceptualization; Data curation; Investigation; Methodology; Supervision; Writing - review \& editing)

\section{Conflict of interest}

None to declare.

\section{References}

1. Scott MC, Winters ME - Congestive Heart Failure. Emerg Med Clin North Am. 2015;33(3):553-62.

2. Ezekowitz JA, McAlister FA, Armstrong PW - Anemia is common in heart failure and is associated with poor outcomes: insights from a cohort of 12065 patients with new-onset heart failure. Circulation. 2003;107(2):223-5.

3. GBD 2016 Disease and Injury Incidence and Prevalence Collaborators - Disease and Injury Incidence and Prevalence Collaborators, Global, regional, and national incidence, prevalence, and years lived with disability for 328 diseases and injuries for 195 countries, 1990-2016: a systematic analysis for the Global Burden of Disease Study 2016 GBD 2016. Lancet. 2017;390(10100):1211-1259.

4. Sundaram V, Fang JC - Gastrointestinal and Liver Issues in Heart Failure. Circulation. 2016;133(17):1696-703.

5. Zain EAS, Mohammad AG, Lobna AW, Elham AH, Khaled MA - Upper Gastrointestinal Mucosal Changes in Patients with Congestive Heart Failure. Med. J. Cairo Univ. 2013;81(1):1009-1014.

6. Romeiro FG, Okoshi K, Zornoff LA, Okoshi MP - Gastrointestinal changes associated to heart failure. Arq Bras Cardiol. 2012;98(3):273-7.

7. van Veldhuisen DJ, Anker SD, Ponikowski P, Macdougall IC - Anemia and iron deficiency in heart failure: mechanisms and therapeutic approaches. Nat Rev Cardiol. 2011;8(9):485-93.

8. Krack A, Sharma R, Figulla HR, Anker SD - The importance of the gastrointestinal system in the pathogenesis of heart failure. Eur Heart $\mathrm{J}$. 2005;26(22):2368-74.

9. Shah R, Agarwal AK - Anemia associated with chronic heart failure: current concepts. Clin Interv Aging. 2013;8:111-22.

10. Lanza FL - Endoscopic Studies of Gastric and Duodenal Injury after the Use of Ibuprofen, Aspirin, and Other Nonsteroidal Anti-Inflammatory Agents. Am J Med. 1984;77(1A):19-24.

11. Raja K, Kochhar R, Sethy PK, Dutta U, Bali HK, Varma JS - An endoscopic study of upperGl mucosal changes in patients with congestive heart failure. Gastrointest Endosc. 2004;60(6):887-93.

12. Sonnenberg A, Genta RM - Changes in the Gastric Mucosa with Aging. Clin Gastroenterol Hepatol. 2015;13(13):2276-81.

13. Jamkhande PG, Gattani SG, Farhat SA - Helicobacter pylori and cardiovascular complications: a mechanism based review on role of Helicobacter pylori in cardiovascular diseases. Integr Med Res. 2016;5(4):244-249.

14. Anand IS, Gupta P - Anemia and Iron Deficiency in Heart Failure: Current Concepts and Emerging Therapies. Circulation. 2018;138:80-98.

15. Arora NP, Ghali JK - Anemia and Iron Deficiency in Heart Failure. Heart Failure Clin. 2014;10(2):281-94.

16. Klip IT, Comin-Colet J, Voors AA, et al - Iron deficiency in chronic heart failure: an international pooled analysis. Am Heart J. 2013;165(4):575582.e3.

17. Negovan A, lancu M, Moldovan V, et al - The contribution of clinical and pathological predisposing factors to severe gastro-duodenal lesions in patients with long-term low-dose aspirin and proton pump inhibitor therapy. Eur J Intern Med. 2017;44:62-66.

18. Stein J, Connor S, Virgin G, Ong DE, Pereyra L - Anemia and iron deficiency in gastrointestinal and liver conditions. World J Gastroenterol. 2016;22(35):7908-25.

19. Negovan A, lancu M, Moldovan V, Mocan S, Banescu C - The Interaction between GST11, GSTM1, and GSTP1 lle105Val Gene Polymorphisms and Environmental Risk Factors in Premalignant Gastric Lesions Risk. Biomed Res Int. 2017;2017:7365080.

20. Negovan A, lancu M, Moldovan V, et al - Influence of MDR1 C3435T, CYP2C19*2 and CYP2C19*3 gene polymorphisms and clinical characteristics on the severity of gastric lesions: a case-control study. J Gastrointestin Liver Dis. 2016;25(2):258-60.

21. Annibale B, Capurso G, Chistolini A, et al - Gastrointestinal causes of refractory iron deficiency anemia in patients without gastrointestinal symptoms. Am J Med. 2001;111(6):439-45.

22. Negovan A, lancu M, Moldovan V,et al - Clinical Risk Factors for 
Gastroduodenal Ulcer in Romanian Low-Dose Aspirin Consumers. Gastroenterol Res Pract. 2016;2016:7230626.

23. Valkhoff VE, Sturkenboom MC, Kuipers EJ - Risk factors for gastrointestinal bleeding associated with low-dose aspirin. Best Pract Res Clin Gastroenterol. 2012;26(2):125-40.

24. García-Rayado G, Sostres C, Lanas A - Aspirin and Omeprazole for Secondary Prevention of Cardiovascular Disease in Patients at Risk for Aspirin-associated Gastric Ulcers. Expert Rev Clin Pharmacol. 2017;10(8):875-888.

25. Jousilahti $P$, Vartiainen $E$, Tuomilehto J, Puska P Sex, age, cardiovascular risk factors, and coronary heart disease: a prospective follow-up study of 14786 middle-aged men and women in Finland. Circulation. 1999;99(9):1165-72.

26. Shiotani A, Sakakibara T, Yamanaka Y, et al - Upper gastrointestinal ulcer in Japanese patients taking low-dose aspirin. Journal of Gastroenterology. 2009;44(2):126-31.

27. Wen L - Upper Gastrointestinal Complications and Cardiovascular/ Gastrointestinal Risk Calculator in Patients with Myocardial Infarction
Treated with Aspirin. Chin Med J(Engl). 2017;130(16):1909-1913.

28. Nema H, Kato M, Katsurada $T$, et al - Endoscopic survey of lowdose-aspirin-induced gastroduodenal mucosal injuries in patients with ischemic heart disease. J Gastroenterol Hepatol. 2008;23 Suppl 2:S234-6.

29. van Deursen VM, Urso R, Laroche C, et al - Co-morbidities in patients with heart failure: an analysis of the European Heart Failure Pilot Survey. Eur J Heart Fail. 2014;16(1):103-11.

30. Serrano P, Lanas A, Arroyo MT, Ferreira IJ - Risk of upper gastrointesninal bleeding in patients taking low-dose aspirin for the prevention of cardiovascular diseases. Aliment Pharmacol Ther. 2002;16(11):1945-53.

31. Bardach AE, Caporale JE, Rubinstein AL, Danaei G - Impact of level and patterns of alcohol drinking on coronary heart disease and stroke burden in Argentina. PloS One. 2017;12(3):e017704.

32. Klatsky AL - Alcohol and cardiovascular diseases. Expert Rev Cardiovasc Ther. 2009;7(5):499-506. 